\title{
RDW-based clinical score to predict long-term survival in community-acquired pneumonia: a European derivation and validation study
}

\author{
Remo Melchio ${ }^{1}$ - Jacopo Davide Giamello ${ }^{1}$ Elisa Testa ${ }^{1}$. Luis Alberto Ruiz Iturriaga ${ }^{2}$. Andrea Falcetta ${ }^{1}$. \\ Cristina Serraino ${ }^{1} \cdot$ Piero Riva $^{3}$. Christian Bracco ${ }^{1} \cdot$ Leyre Serrano Fernandez $^{2}$. Salvatore D'Agnano ${ }^{1}$. \\ Stefano Leccardi ${ }^{1} \cdot$ Massimo Porta $^{3} \cdot$ Luigi Maria Fenoglio $^{1}$
}

Received: 3 September 2020 / Accepted: 18 December 2020 / Published online: 11 January 2021

(c) Società Italiana di Medicina Interna (SIMI) 2021

\begin{abstract}
An excess long-term mortality has been observed in patients who were discharged after a community-acquired pneumonia (CAP), even after adjusting for age and comorbidities. We aimed to derive and validate a clinical score to predict long-term mortality in patients with CAP discharged from a general ward. In this retrospective observational study, we derived a clinical risk score from 315 CAP patients discharged from the Internal Medicine ward of Cuneo Hospital, Italy, in 2015-2016 (derivation cohort), which was validated in a cohort of 276 patients discharged from the pneumology service of the Barakaldo Hospital, Spain, from 2015 to 2017, and from two internal medicine wards at the Turin University and Cuneo Hospital, Italy, in 2017. The main outcome was the 18-month follow-up all-cause death. Cox multivariate analysis was used to identify the predictive variables and develop the clinical risk score in the derivation cohort, which we applied in the validation cohort. In the derivation cohort (median age: 79 years, $54 \%$ males, median CURB-65 =2), 18-month mortality was 32\%, and $18 \%$ in the validation cohort (median age 76 years, $55 \%$ males, median CURB-65 $=2$ ). Cox multivariate analysis identified the red blood cell distribution width (RDW), temperature, altered mental status, and Charlson Comorbidity Index as independent predictors. The derived score showed good discrimination (c-index $0.76,95 \%$ CI $0.70-0.81$; and 0.83 , 95\% CI 0.78-0.87, in the derivation and validation cohort, respectively), and calibration. We derived and validated a simple clinical score including RDW, to predict long-term mortality in patients discharged for CAP from a general ward.
\end{abstract}

Keywords Community-acquired pneumonia $\cdot$ Prognosis $\cdot$ Red blood cell distribution width $\cdot$ Clinical score

Supplementary Information The online version contains supplementary material available at https://doi.org/10.1007/s1173 9-020-02615-6.

Remo Melchio

remo.melchio@libero.it

1 Department of Internal Medicine, A.O. S. Croce e Carle, Via Michele Coppino 26, 12100 Cuneo, CN, Italy

2 Pneumology Service, Hospital Universitario Cruces, Barakaldo, Bizkaia, Spain

3 Department of Medical Sciences, University of Turin - AOU Città della Salute e della Scienza di Torino, Turin, Italy

\section{Introduction}

Community-acquired pneumonia (CAP) remain a major cause of morbidity and mortality worldwide [1]. Although early (e.g. in-hospital and 30-day mortality) is estimated to be $10 \%$ for patients admitted to a general ward [2], CAP has a strong impact on long-term prognosis ( $>3$ months). An increase in the death risk in CAP patients who were discharged from the hospital was as high as $50 \%$ in a 5-year follow-up, even after adjusting for age and comorbidities [3-5]. Thus, acute diseases such as pneumonia show an excess long-term mortality, comparable to that of the most common chronic conditions (e.g. cancer, heart failure). The ability to identify the patients at high risk of adverse outcome in an adequate timespan after the discharge from a medical ward for an episode of CAP remains challenging. Nevertheless, the availability of a bedside clinical prediction 
score could improve the home care management, follow-up organization and communication with patients and their relatives about prognosis.

A series of risk factors have been shown to be linked to long-term mortality in CAP, including elderly age, male sex, and chronic comorbidities [6, 7]. A well known and validated method for classifying comorbid conditions, the Charlson Comorbidity Index (CCI), when studied in CAP, has shown a poor accuracy as a standalone method for predicting 1-year mortality [8,9]. Moreover, various biomarkers as pro-adrenomedullin, copeptin, endhotelin-1, procalcitonin, and C-reactive protein have been associated with long-term prognosis [10-12]. Although some of these predictors are easy to obtain in clinical practice, others are more complex or expensive to acquire, limiting their widespread use at the bedside [13]. Red blood cell distribution width (RDW), a measure of anisocytosis, is a parameter that routinely reported in the complete blood cell counts, and, therefore, virtually available to all patients at no cost. Recently, several studies have evaluated its prognostic accuracy in various diseases [13-17], including pneumonia [18, 19]. Although high RDW values have been associated with unfavorable short and long-term prognosis in CAP, no multivariable model has been done so far.

Therefore, in this study, we used clinical and laboratory data (including RDW) from three European hospitals to derive and validate a risk prediction score, to estimate the 18-month mortality in patients who experienced an episode of CAP and were discharged from a general ward.

\section{Methods}

\section{Study design and patients}

We followed the TRIPOD [20] (Transparent Reporting of a Multivariable Prediction Model for Individual Prognosis or Diagnosis) statement for reporting multivariable prediction model development and validation. This study complied with the Declaration of Helsinki and was approved by the local ethics committees.

The derivation cohort consisted of all consecutive patients discharged from the Internal Medicine Department of the Santa Croce and Carle Hospital of Cuneo from 1 January 2015 through 31 December 2016 with a main diagnosis of 'Community-Acquired Pneumonia'. The Cuneo Hospital is a tertiary 650-bed teaching hospital, with 25,000 admissions in 2016, located in the southern Piedmont region, Italy. We selected the patients by searching the administrative database of the hospital with the following discharge codes, according to the International Classification of the Diseases (ICD)-9: 480 to 487.0. Each retrieved patient record was checked by JDG and CB to verify the inclusion and exclusion criteria.

Patients were diagnosed with CAP, if they had an acute illness with cough and at least one of new focal chest signs, fever $>4$ days or dyspnoea/tachypnoea, without other obvious causes, supported by chest radiograph findings of the lung shadowing that were likely to be new, according to the European Respiratory Society Guidelines [21].

The exclusion criteria were (1) patients who died or were missing within the first 30 days of admission; (2) severe immunodepression (HIV infection, severe haematological diseases or immunosuppressive therapy; (3) leukopenia $\left(<1000\right.$ leukocyte per $\left.\mathrm{mm}^{3}\right)$ or neutropoenia $(<500$ neutrophils per $\mathrm{mm}^{3}$ ); (4) metastatic cancer; (5) pulmonary abscess or aspiration pneumonia; (6) possible nosocomial origin ( $<30$ days from hospital discharge); and (7) blood transfusion in the previous three months. In patients with multiple admissions, only the index admission was considered.

Patients were treated according to the guidelines of the European Respiratory Society (ERS) and the European Society for Clinical Microbiology and Infectious Diseases (ESCMID) [21].

The derivation cohort included 315 patients (Supplemental Material Figure S1).

The external validation cohort was composed of 276 patients with CAP discharged in part from the Pneumology Service of the Hospital Universitario Cruces, Barakaldo in Bilbao, Spain from 2015 to $2017(n=96)$ and in part from the Internal Medicine ward of the Città della Salute e della Scienza University Hospital of Turin (Italy) in 2016 $(n=114)$, and from the Internal Medicine ward of the Santa Croce and Carle Hospital of Cuneo (Italy) in $2017(n=80)$, (Supplemental Material Figure S1). The Spanish cohort is based on a prospective registry of consecutive immunocompetent adults ( $>18$ years) hospitalized for communityacquired pneumococcal pneumonia in Cruces Hospital [4]. For Italian cohort, inclusion and exclusion criteria were the same as in the derivation cohort.

\section{Data collection}

For each patient in the derivation cohort, demographic, clinical, laboratory, and radiological variables data were collected by consulting the records of the emergency departments, electronic medical records, and discharge summaries.

The variables collected included: demographic characteristics (sex and age), underlying diseases and comorbidities, residence in nursing home, dialysis, smoking, and home oxygen therapy. The Charlson Comorbidity Index, a weighted measure of comorbidities, was also calculated [9] (Supplemental Material, Figure S2).

Vital parameters, altered mental status (Abbreviated Mental Test < 8) [22] and laboratory data at admission were 
also collected, glomerular filtration rate was estimated using the CKD-EPI formula [23]. RDW was reported as the coefficient of variation (in percent) of erythrocyte cell volume, and the blood count data were obtained using the Dasit Sysmex XE-5000 instrument. For each patient data on blood cultures, sputum, bronchoalveolar lavage (BAL), urinary legionella, and pneumococcal antigens were collected when available. Chest radiographs were examined to evaluate the bilateral involvement. Data on antibiotic therapy were also collected, reporting the class of drug used, the associations, and any changes in the treatment. Moreover, the Pneumonia Severity Index (PSI) [24] and CURB-65 [25], severity scores were calculated.

\section{Study outcome}

For both the derivation and validation cohorts, the main outcome was all-cause mortality at 18 months: this timespan was chosen for clinical convenience, and because it was evaluated in previous studies on CAP prognosis $[11,26]$. Follow-up was performed by consulting the hospital information database connected to the Regional Registry Office or by phone calls to patients, to their relatives or to the referring primary physicians.

To assess the quality of follow up, we calculated the simplified person-time follow-up rate [27], which was 93\% in the derivation cohort and $92 \%$ in the validation cohort.

\section{Statistical analysis}

Continuous variables with normal distribution are presented as mean \pm standard deviation, whereas those with non-normal distribution are presented as median and interquartile range. Categorical variables are presented as frequencies and percentages.

The sample size was calculated using the conservative rule of 10 events per candidate predictor: in the derivation cohort, 100 events allowed almost 10 candidate variables to be included in the multivariable Cox model. In the validation cohort, we needed almost 40 events to validate the 4 -variables prediction model.

Survival curves were generated using the Kaplan-Meier method and compared with the log-rank test. In the derivation cohort, univariate and multivariate Cox proportional hazard models were used to identify the variables associated with survival. Only variables that reached a statistical significance $(\mathrm{p}<0.05)$ in the univariate analysis were included in the full multivariate Cox models and were subjected to a stepwise multivariate Cox analysis.

We tested both a model with single comorbidities and age as covariates and a model with Charlson Comorbidities Index (a weighted score of comorbidities and age). Similarly, a model including PSI classes was evaluated along with haemoglobin, RDW, eGFR, and C-reactive protein. The variables included in the final model after stepwise selection analysis were the Charlson Comorbidity Index, altered mental status, RDW, and temperature. Then, to estimate more easily the individual risk at the bedside, we generated a simplified score by dividing the $\beta$ coefficients for each category by the smallest value of a $\beta$ coefficient in the model to allocate an integer score for each variable [28]. The resulting derivative score was finally validated at the multicentre validation cohort.

As the simplification of the developed prediction model could have led to some loss of predictive accuracy, to assess the discriminatory power (i.e. the ability of the model to differentiate between those who do or do not experience the outcome event), we determined the area under the receiveroperating curve (AUC) for both the prediction model and the simplified final score in derivation and validation cohorts.

Model calibration (i.e. the agreement between predictions from the model and observed outcomes) was assessed by the calibration intercept and the calibration slope, and graphically by comparing observed (Kaplan-Meier) and predicted survival probabilities in three prognostic groups (low, medium, and high risk) derived by placing cut-points on the prognostic index [29,30].

All tests were two-sided and statistical significance was accepted, if the $\mathrm{P}$ value was $<0.05$.

All statistical analyses were conducted using STATA/IC 14.0 software (StataCorp LP, USA).

\section{Results}

\section{Derivation cohort}

The clinical and laboratory characteristics of the derivation cohort are reported in Table 1.

The median age was 79 years, $54 \%$ were men, and the 18 -month mortality rate was $32 \%$. The main comorbidities were heart failure (19\%), diabetes mellitus (20\%), cerebrovascular disease (20\%), non-metastatic cancer (20\%), and COPD (18\%). The median Charlson comorbidity index was 6 (IQR 4-8). The median value of CURB-65 was 2, and 66\% of the population belonged to PSI class IV to V. The median RDW value at admission was $14.4 \%$ (95\% CI 13.6-15.9). The most used antibiotics were beta-lactams and macrolides, administered to $62 \%$ of patients, followed by fluoroquinolones, administered to $37 \%$ of patients.

\section{Validation cohort}

The general characteristics of the validation cohort are shown in Table 1. Compared with the derivation cohort, patients were younger (median age: 76 years), had fewer 
Table 1 Baseline characteristics of the derivation and validation cohorts
Cohort, no. (\%) of patients

Derivation $(N=315)$

Validation $(N=276)$

Demographics

Age, median (IQR), year

Gender (women)

$79(70-85)$

$76(61-83)$

$143(45)$

$124(45)$

Nursing home resident

$74(23)$

$14(5)$

Comorbidities

Coronary artery disease

$40(13)$

34 (12)

Heart failure

$59(19)$

$33(12)$

Diabetes mellitus

Cerebrovascular disease

$64(20)$

$61(22)$

$65(20)$

$51(18)$

Dementia

COPD

$38(12)$

25 (9)

$58(18)$

54 (20)

Cancer

$64(20)$

37 (13)

Chronic kidney disease $(\mathrm{eGFR}<30)$

$42(13)$

24 (9)

Liver disease

Charlson Comorbidity Index

$15(5)$

$9(4)$

$6(4-8)$

$4(2-6)$

Number of comorbidities

0

$38(12)$

$85(30)$

$68(21)$

69 (25)

$>1$

Active smoker

209 (66)

$122(44)$

28 (18)

67 (24)

Severity of Pneumonia

CURB-65 score

PSI score

PSI class I-II

$2(1-2)$

$2(1-2)$

$102(84-128)$

$93(73-114)$

39 (12)

$70(25)$

66 (21)

$72(26)$

IV

134 (42)

99 (36)

75 (24)

$32(12)$

Vital parameters at admission

Systolic blood pressure (mmHg)

Heart rate (bpm)

Respiratory rate (median, IQR)

Body temperature (median (IQR), ${ }^{\circ} \mathrm{C}$ )

Arterial oxygen saturation (\%)

Altered mental status at admission (\%)

$133 \pm 22$

$127 \pm 23$

$92+18$

$94 \pm 20$

$16(14-20)$

20 (16-24)

$37.7(36.8-38.5)$

$37.3(36.5-38.2)$

93 (90-96)

92 (88-95)

67 (21)

35 (13)

Bilateral involvement at $\mathrm{Rx}(\%)$

$65(21)$

65 (23)

Laboratory data

Haemoglobin $(\mathrm{g} / \mathrm{L})$

Haematocrit (median (IQR), \%)

$12.5 \pm 1.9$

$12.7 \pm 1.9$

$37.6(34-41)$

$38.2(35-42)$

RDW (median (IQR), \%)

$14.4(13.6-15.9)$

$14.2(13.4-15.4)$

GFR $(\mathrm{ml} / \mathrm{min})$

$65.2+28$

$67.9 \pm 28$

BUN (median (IQR), mg/dL)

C-Reactive Protein (median (IQR), mg/dL)

$23(16-33)$

$22(15-32)$

$68(33-191)$

$0.27(0.12-1.3)$

$43(21-125)$

Procalcitonin $(\mathrm{mg} / \mathrm{L})$

$138.3(135-141)$

$0.20(0.1-1.0)^{*}$

$137(134-139)$

$128(109-152)$

Glucose $(\mathrm{mg} / \mathrm{dL})$

$102(32)$

$51(18)$

Data are expressed as number (percentage), mean \pm standard deviation or median $(25-75 \%$ percentiles) as appropriate

$B U N$ blood urea nitrogen, $C O P D$ chronic obstructive pulmonary disease, $e G F R$ estimated glomerular filtration rate, $P S I$ Pneumonia Severity Index, $R D W$ red cell distribution width

*Data available for $N=187$ 
comorbidities (55\% had 1 or no comorbidities), and belonged to a PSI class IV or V in $48 \%$ of cases. The median CURB-65 score was 2, similar to that of the derivation cohort. The median RDW value at admission was $14.2 \%$ (95\% CI 13.4-15.4) (Table 1). The 18-month mortality rate was $18 \%$ (51 patients). Based on the available data, the most used antibiotics were beta-lactams and macrolides, administered to $77 \%$ of the patients, followed by fluoroquinolones, administered to $22 \%$ of patients.

\section{Univariate analysis}

The univariate analysis of the derivation cohort (Table 2), identified the variables associated with the main outcome. For each year increase in age, the risk of death increased by $3 \%$ (95\% CI 1-5\%). Among the comorbidities, heart failure, dementia, chronic kidney disease, and chronic liver disease were associated with an increased risk of 18-month mortality. For every point increase of the Charlson Comorbidity Index, the incremental mortality risk was $21 \%$. In patients presenting in the ED with altered mental status, the risk of death at 18 months tripled (HR 3.2, 95\% CI 2.1-4.8), increased body temperature was associated with reduced mortality by $25 \%$ for each additional degree (HR $0.75,95 \%$ CI 0.62-0.92). Among the laboratory variables, haemoglobin, RDW, eGFR, BUN, and C-reactive protein were associated with the survival. Specifically, for every additional percentage increase in RDW value at the admission, 18-month mortality increased by $18 \%$ (95\% CI 9-28\%) and an RDW greater than or equal to the median value (14.4\%) almost doubles the probability of death during follow-up (HR 1.89, 95\% CI 1.39-2.56). The severity of pneumonia, assessed by the CURB-65 score or PSI classes, was also strongly associated with the long-term survival.

\section{Multivariate analysis and score validation}

Variables that reached statistical significance $(p<0.05)$ in the univariate analysis were included in the full multivariate Cox model and were subjected to a stepwise multivariate Cox regression analysis. To avoid duplicate covariate insertion, we tested both a model with single comorbidities and age as covariates and a model with the Charlson Comorbidities Index (a weighted score of comorbidities and age). For the same reason, a model including PSI classes was evaluated along with haemoglobin, RDW, eGFR, and C-reactive protein.

The variables included in the final Cox model after stepwise selection analysis were the Charlson Comorbidity Index, altered mental status, RDW, and temperature (Table 3, Model 2).

Discrimination was evaluated by calculating the area under the receiver operating curve (AUC) of the
Table 2 Univariate analysis of risk factors associated with 18-month mortality in the derivation cohort $(N=315)$

\begin{tabular}{|c|c|c|c|}
\hline & HR & $95 \% \mathrm{CI}$ & $p$ value \\
\hline \multicolumn{4}{|l|}{ Demographics } \\
\hline Age, years & 1.03 & $1.01-1.05$ & 0.005 \\
\hline Gender (women) & 0.97 & $0.65-1.44$ & 0.87 \\
\hline Nursing home resident & 1.35 & $0.87-2.10$ & 0.17 \\
\hline \multicolumn{4}{|l|}{ Comorbidities } \\
\hline Coronary artery disease & 1.33 & $0.76-2.30$ & 0.30 \\
\hline Heart failure & 1.91 & $1.23-2.96$ & 0.004 \\
\hline Diabetes mellitus & 1.15 & $0.72-1.85$ & 0.54 \\
\hline Cerebrovascular disease & 1.03 & $0.64-1.67$ & 0.90 \\
\hline Dementia & 2.97 & $1.87-4.71$ & 0.000 \\
\hline COPD & 1.14 & $0.70-1.85$ & 0.57 \\
\hline Cancer & 1.38 & $0.88-2.16$ & 0.15 \\
\hline Chronic kidney disease & 1.21 & $0.99-1.47$ & 0.06 \\
\hline Liver disease & 1.59 & $1.21-2.08$ & 0.001 \\
\hline Charlson comorbidity score & 1.21 & $1.11-1.32$ & 0.000 \\
\hline Active smoker & 1.35 & $0.67-2.73$ & 0.85 \\
\hline \multicolumn{4}{|l|}{ Severity of pneumonia } \\
\hline CURB-65 & 1.86 & $1.42-2.41$ & 0.000 \\
\hline PSI class & 1.77 & $1.40-2.25$ & 0.000 \\
\hline \multicolumn{4}{|l|}{ Vital parameters at admission } \\
\hline Systolic blood pressure (mmHg) & 0.99 & $0.99-1.00$ & 0.94 \\
\hline Heart rate $(\mathrm{bpm})$ & 1.00 & $0.99-1.01$ & 0.10 \\
\hline Respiratory rate & 1.04 & $0.99-1.09$ & 0.08 \\
\hline Body temperature $\left({ }^{\circ} \mathrm{C}\right)$ & 0.75 & $0.62-0.92$ & 0.006 \\
\hline Arterial oxygen saturation (\%) & 0.98 & $0.93-1.02$ & 0.25 \\
\hline $\begin{array}{l}\text { Altered mental status at admission } \\
(\%)\end{array}$ & 3.23 & $2.16-4.82$ & 0.000 \\
\hline Bilateral involvement at $\mathrm{Rx}(\%)$ & 1.03 & $0.65-1.65$ & 0.88 \\
\hline \multicolumn{4}{|l|}{ Laboratory data at admission } \\
\hline Haemoglobin (g/L) & 0.85 & $0.77-0.95$ & 0.006 \\
\hline Haematocrit (\%) & 0.98 & $0.95-1.03$ & 0.42 \\
\hline RDW (\%) & 1.18 & $1.09-1.28$ & 0.000 \\
\hline eGFR (ml/min) & 0.991 & $0.984-0.998$ & 0.01 \\
\hline BUN (mg/dL) & 1.02 & $1.01-1.03$ & 0.000 \\
\hline C-reactive protein (mg/dL) & 0.997 & $0.996-0.999$ & 0.03 \\
\hline Procalcitonin (mg/L) & 0.984 & $0.951-1.017$ & 0.34 \\
\hline Sodium (mEq/L) & 1.02 & $0.99-1.05$ & 0.15 \\
\hline Glucose (mg/dL) & 1.00 & $0.99-1.00$ & 0.32 \\
\hline
\end{tabular}

Data are expressed as Number (Percentage), mean \pm standard deviation or median (25-75\% percentiles) as appropriate

$B U N$ Blood Urea Nitrogen, COPD chronic obstructive pulmonary disease, $e G F R$ estimated glomerular filtration rate, PSI Pneumonia Severity Index, $R D W$ red cell distribution width

*Data available for $\mathrm{N}=283$

prediction model in the derivation (c-statistic $0.77,95 \%$ CI $0.72-0.82$ ) and validation cohorts (c-statistic $0.85,95 \%$ CI 0.80-0.89) (Fig. 1). Model calibration was assessed by the calibration intercept and the calibration slope, and 
Table 3 Cox multivariable models for prediction of 18 -month mortality in the derivation cohort $(n=315)$

\begin{tabular}{|c|c|c|c|c|c|}
\hline & Variable & Beta & HR & $95 \%$ CI & $p$ value \\
\hline \multicolumn{6}{|l|}{ Model 1} \\
\hline & Heart failure (yes/no) & 0.69 & 2.00 & $1.25-3.18$ & 0.003 \\
\hline & CKD (yes/no) & 0.65 & 1.91 & $1.12-3.30$ & 0.01 \\
\hline & Liver disease (yes/no) & 1.24 & 3.47 & $1.64-7.37$ & 0.001 \\
\hline & Dementia & 0.68 & 1.98 & $1.14-3.44$ & 0.01 \\
\hline & Altered mental status (yes/no) & 0.92 & 2.51 & $1.54-4.09$ & 0.000 \\
\hline & RDW (\%) & 0.17 & 1.19 & $1.08-1.30$ & 0.000 \\
\hline & Temperature $\left({ }^{\circ} \mathrm{C}\right)$ & -0.23 & 0.79 & $0.65-0.96$ & 0.02 \\
\hline \multicolumn{6}{|l|}{ Model 2} \\
\hline & $\begin{array}{l}\text { Charlson Comorbidity Index (every } \\
1 \text { point) }\end{array}$ & 0.18 & 1.19 & $1.09-1.31$ & 0.000 \\
\hline & Altered mental status (yes/no) & 1.04 & 2.83 & $1.87-4.27$ & 0.000 \\
\hline & $\mathrm{RDW}(\%)$ & 0.15 & 1.17 & $1.07-1.27$ & 0.001 \\
\hline & Temperature $\left({ }^{\circ} \mathrm{C}\right)$ & -0.20 & 0.81 & $0.67-0.99$ & 0.04 \\
\hline \multicolumn{6}{|l|}{ Model 3} \\
\hline & PSI class (every 1 class, I to V) & 0.61 & 1.85 & $1.42-2.40$ & 0.000 \\
\hline & RDW (\%) & 0.17 & 1.18 & $1.08-1.30$ & 0.000 \\
\hline & Temperature $\left({ }^{\circ} \mathrm{C}\right)$ & -0.27 & 0.76 & $0.63-0.92$ & 0.006 \\
\hline
\end{tabular}

$C K D$ chronic kidney disease, $P S I$ Pneumonia Severity Index; $R D W$ red cell distribution width
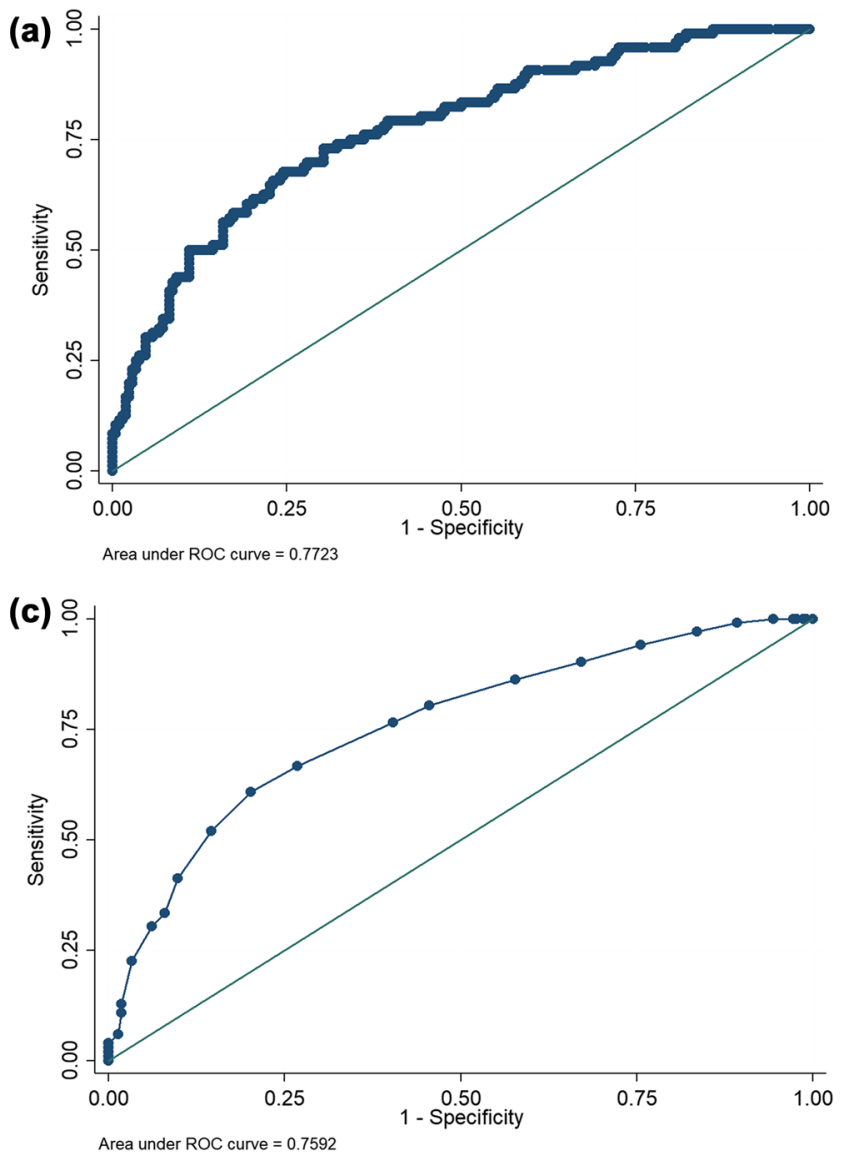
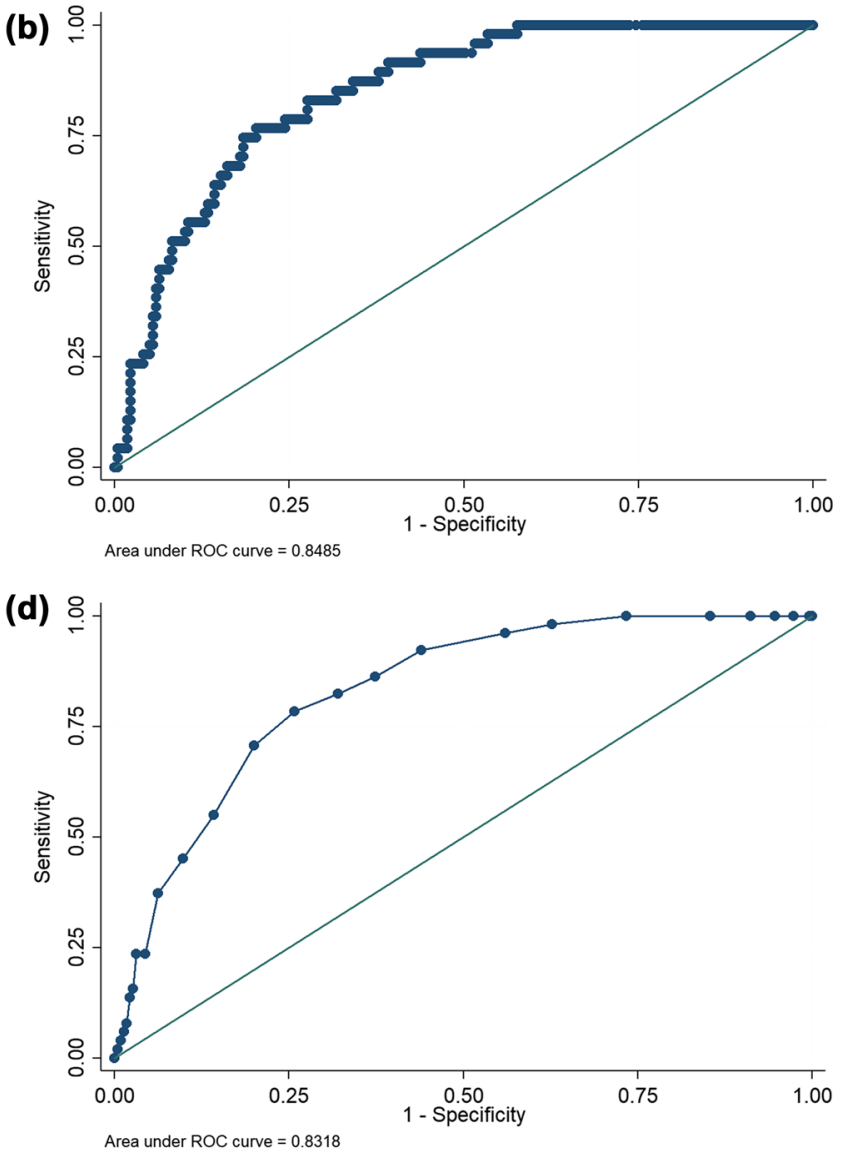

Fig. 1 Performance (c-statistic) of the final model and the simplified score. Top: AUC of the final model in the derivation (a) and validation (b) cohorts. Bottom: AUC of the score in the derivation (c) and validation (d) cohorts 
graphically by comparing observed (Kaplan-Meier) and predicted survival probabilities in three prognostic groups (low, medium, and high risk) derived by placing cut-points on the 25th and 75th percentile of the prognostic index (Fig. 2). We then divided the $\beta$ coefficients for each category by the smallest value of a $\beta$ coefficient in the derivation model, to allocate an integer score for each variable (Table 4). The resulting derivative score was finally validated in the external validation cohort, and measures of discrimination and calibration were calculated accordingly (Figs. 1, 2, and 3). The score showed an overall good accuracy as expressed by the c-statistic $=0.83(95 \% \mathrm{CI}$ $0.78-0.87$ ) and by the calibration slope $=1.09$ (Fig. 3) Adding RDW significantly improved the prediction score in the derivation (AUC $=0.67,95 \%$ CI $0.60-0.73$, without RDW; vs $\mathrm{AUC}=0.76,95 \%$ CI $0.70-0.81$, with RDW; $\mathrm{p}=0.001)$ and in the validation cohort $(\mathrm{AUC}=0.78,95 \%$ CI $0.72-0.84$, without $\mathrm{RDW}$; vs $\mathrm{AUC}=0.83,95 \%$ CI $0.78-0.87$, with RDW; $\mathrm{p}=0.05$; Supplemental Material, Figure S3).

\section{Discussion}

In this study involving more than 500 patients who were discharged after an episode of CAP from three European hospitals, we derived and validated a simple risk-prediction score to estimate the risk of 18-month mortality.

The predictors that were included in the final multivariate model and used to compose the score were age and comorbidities (summarized and weighted in the Charlson Comorbidity Index), altered mental status, temperature, and RDW at admission.

Several studies have assessed the association between comorbidities, age, biomarkers, severity of pneumonia, and long-term mortality in patients with CAP [4-8, 10, 11].

Guerter et al. in a derivation study including 877 patients with CAP, enrolled in the multicenter ProHOSP trial showed that male sex, age, comorbidities (COPD and cancer), low temperature, low CRP and high pro-adrenomedullin values were all independently associated with 18-month mortality [11]. Although the derived score has not yet been validated,
Fig. 2 Kaplan-Meier estimated the event-free survival rates per risk stratum in the derivation (a) and validation (b) cohorts. Observed and predicted survival probabilities in three prognostic groups (low, medium and high risk) were 2 derived by placing the cut points on the 25 th and 75 th percentiles of the prognostic index
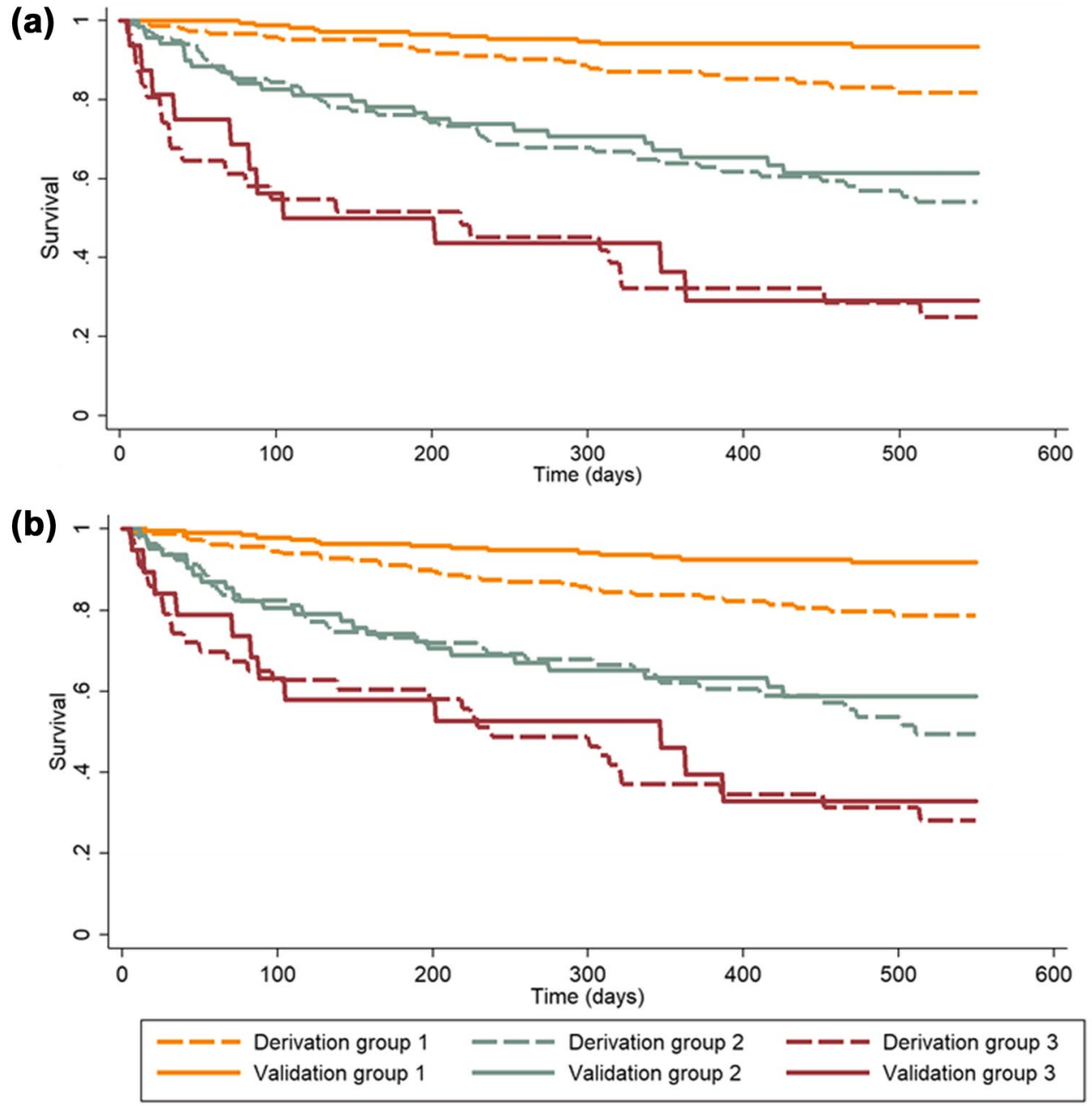
Table 4 18-month mortality risk index for patients with CAP

\begin{tabular}{|c|c|}
\hline RDW (\%) & Points \\
\hline $12-13$ & 0 \\
\hline $13-14$ & 1 \\
\hline $14-15$ & 2 \\
\hline $15-16$ & 3 \\
\hline $16-17$ & 4 \\
\hline $17-18$ & 5 \\
\hline $18-19$ & 6 \\
\hline $19-$ & 7 \\
\hline Temperature $\left({ }^{\circ} \mathrm{C}\right)$ & Points \\
\hline$<35$ & 5 \\
\hline $35-35.9$ & 4 \\
\hline $36-36.9$ & 3 \\
\hline $37-37.9$ & 2 \\
\hline $38-38.9$ & 1 \\
\hline $39-$ & 0 \\
\hline Altered mental status & Points \\
\hline Yes & 6 \\
\hline No & 0 \\
\hline Charlson Comorbidity Index & Points \\
\hline Total risk score & $=()$ \\
\hline
\end{tabular}

Point values for each variable (A patient's total risk score can be obtained by summing points assigned to values of each variable, which can determine his/her corresponding predicted 18-month mortality risk)

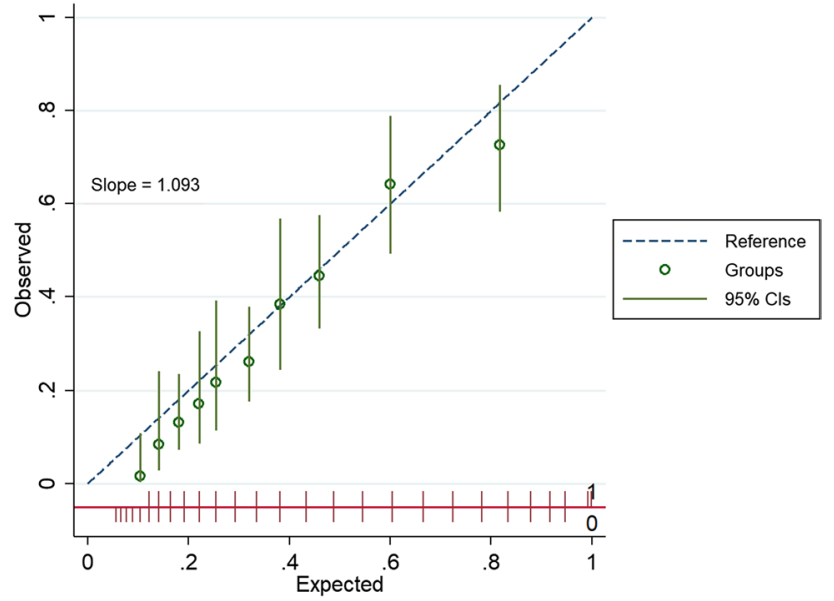

Fig. 3 Calibration plot comparing 18-month mortality risk in derivation and validation cohorts for patients discharged from a general ward after an admission for CAP

several variables, including comorbidities, age, and low temperature are also present in our model. Our study confirms the association between low temperature, CRP values, and worse outcome, a finding possibly ascribed to a reduced immune and inflammatory response in the elderly population. Age-associated alterations in immune surveillance and dysfunctional immune response regulation can be responsible for increased susceptibility to infectious stimuli, and hence, a poor prognosis [31, 32]. Moreover, as expected in our older population, a model including single comorbidities and age or including the Charlson Comorbidity Index, showed a similar prognostic accuracy in the derivation cohort.

In addition, the results of our study confirm the strong ability of RDW in predicting long-term mortality in patients who were discharged for CAP from a general ward: an RDW $>14.4 \%$ (the median value of our population) almost doubles the probability of death at 18 months. Moreover, our data confirm the previous findings showing that its prognostic value is independent of haemoglobin values and comorbidities [13]. Furthermore, our analysis showed a significant improvement in the prediction accuracy when RDW was added to the clinical score, as assessed by comparing the AUCs when calculated with or without this covariate in both the cohorts. Several studies have associated RDW with short-term outcomes in CAP, such as complicated hospitalization, intra-hospital mortality, 30-day mortality and the need to switch to intensive care [33-35]. Torres first studied RDW (comparing it with pro-adrenomedullin) on 
longer-lasting follow-up, showing a good long-term predictive power in CAP [18], as our data confirms.

The pathophysiological mechanism linking RDW with mortality is not yet understood. It has been suggested that anisocytosis could be a marker of cellular aging, inflammation, oxidative stress, hypoxia, splenic immune dysregulation or poor nutritional status, all associated with erythropoiesis or hemocatheresis abnormalities $[16,36]$. However, a high RDW could also contribute to a worse prognosis, because less deformable erythrocytes could impair tissue oxygenation [14]. Moreover, Corrales et al. have suggested an increased risk of cardiovascular disease during and after serious infections, such as pneumonia, and it is suggested that cardiovascular events may play an important role in long-term outcomes [37, 38]. Since RDW has been associated with cardiovascular mortality in patients with and without pre-existing heart diseases [39, 40], one could speculate that our multivariable prediction model could have intercepted a portion of the outcome linked to cardiovascular death.

As in the derivation cohort the $23 \%$ of patients were nursing home residents, one could argue that the inclusion of nursing home-acquired pneumonia, a subset of the healthcare-associated pneumonia (HCAP), could have affected our results. However, it is debated whether to consider HCAP, as a distinct clinical entity in Europe [41]. As summarized in the International ERS/ESICM/ESCMID/ALAT guidelines for the management of hospital-acquired pneumonia and ventilator-associated pneumonia, studies in the USA have reported that HCAP is often caused by multidrug-resistant microorganisms in critically ill patients. In contrast, European data [42] suggest that the aetiology in HCAP patients is similar to that of CAP and that these patients are often not critically ill [43]. In addition, some authors suggest that comorbidities, dementia, aspiration pneumonia, functional impairment and recurrent hospitalization, are probably more significant issues in predicting long-term prognosis for patients with pneumonia acquired in a nursing home [41]. In our study we addressed these variables, excluding patients with aspiration pneumonia, and recurrent hospitalization, and including the comorbidities in the final model. This patient selection method could explain the lack of association of the category 'nursing home residents' with mortality in the univariate analysis in our study.

To confirm this hypothesis, we performed a secondary analysis in the derivation cohort after removing the nursing home residents from the analysis (Supplemental Material Table S1). In this new population $(N=241$, median age: 75 years, $25-75$ percentile $70-84), 18$-month mortality was $30 \%$, and univariate analysis confirmed the associations observed in the primary analysis. Multivariate analysis showed that RDW, altered mental status, and
Charlson comorbidity index all maintained the predictive value found in the primary analysis, however, the temperature did not, probably because of the reduced sample size.

Furthermore, the external validation cohort displayed a lower burden of comorbidities, and therefore, as expected, showed a lower mortality rate, compared with the derivation cohort. Despite these important differences, the derived score showed good predictive power, as shown by a good discrimination (accuracy) and calibration (accuracy across a range of predicted probabilities) in the validation cohort.

Finally, the risk score has important characteristics for clinical practice. It is based on the readily available clinical and laboratory values, and provides estimates of longterm mortality, offering clinicians caring for patients with CAP as a simple tool to select high-risk patients, organize follow-up, and eventually, communicate with patients and their relatives about prognosis.

We acknowledge that our study has several limitations. First, it was a retrospective observational study, and derivation analysis was based on data collected in a single institution. However, the variables used in the final model (including RDW) were available in all patients included in the study, avoiding potential selection bias due to an incomplete collection of data typical of a retrospective design. Second, although the prognostic model shows good accuracy in the discrimination and calibration analysis, we are aware that residual confounding could be present. Third, an analysis of cause-specific mortality could not be performed because the causes of death at long-term follow-up could not be consistently identified. Fourth, observational and registry data show that the hospital admission is associated with an increased prevalence of drug interactions and polypharmacy (the use of five or more prescribed drugs at the same time) in older patients, with potential negative prognostic effects [44, 45]. Although polypharmacy could be an appealing variable to be included in a prognostic model for an elderly population with a high comorbidity index, this data is lacking for most of our patients. However, to date, the association between polypharmacy and long-term mortality in CAP has not yet been established.

In conclusion, we derived and validated a clinical score to predict 18 -month mortality in patients who survived after an acute episode of CAP. For the first time, we introduced RDW in a multivariable predictive model including age, comorbidities, altered mental status and temperature at admission. Further studies are needed to evaluate the clinical impact of the score on the follow-up of patients with CAP discharged from a general ward.

Funding None. 


\section{Compliance with ethical standards}

Conflicts of interest The authors declare that they have no conflict of interest.

Ethics approval All procedures performed in studies involving human participants were in accordance with the ethical standards of the institutional and/or national research committee and with the 1964 Helsinki Declaration and its later amendments or comparable ethical standards. The study was approved by the institutional ethic committee.

Consent to participate This is a retrospective study that includes anonymized patients extracted from the hospital database, data are presented aggregated and anonymously. No informed consent was taken.

\section{References}

1. WHO (2016) The top ten causes of death. WHO, Geneva

2. Prina E, Ranzani OT, Torres A (2015) Community-acquired pneumonia. Lancet. 1097-1108

3. Restrepo MI, Faverio P, Anzueto A (2013) Long-term prognosis in community-acquired pneumonia. Curr Opin Infect Dis 26:151-158

4. Ruiz LA, Serrano L, España PP et al (2019) Factors influencing long-term survival after hospitalization with pneumococcal pneumonia. J Infect. https://doi.org/10.1016/j.jinf.2019.10.024

5. Uranga A, Quintana JM, Aguirre U et al (2018) Predicting 1-year mortality after hospitalization for community-acquired pneumonia. PLoS ONE. https://doi.org/10.1371/journal.pone.0192750

6. Bruns AHW, Oosterheert JJ, Cucciolillo MC et al (2011) Causespecific long-term mortality rates in patients recovered from community-acquired pneumonia as compared with the general Dutch population. Clin Microbiol Infect. https://doi.org/10.111 1/j.1469-0691.2010.03296.x

7. Sibila O, Mortensen EM, Anzueto A et al (2014) Prior cardiovascular disease increases long-term mortality in COPD patients with pneumonia. Eur Respir J 43:36-42

8. Wesemann T, Nüllmann H, Nüllmann $\mathrm{H}$ et al (2015) Pneumonia severity, comorbidity and 1-year mortality in predominantly older adults with community-acquired pneumonia: a cohort study. BMC Infect Dis. https://doi.org/10.1186/s12879-014-0730-x

9. Charlson ME, Pompei P, Ales KL, MacKenzie CR (1987) A new method of classifying prognostic comorbidity in longitudinal studies: development and validation. J Chronic Dis 40:373-383. https ://doi.org/10.1016/0021-9681(87)90171-8

10. Krüger S, Ewig S, Giersdorf S et al (2010) Cardiovascular and inflammatory biomarkers to predict short- and long-term survival in community-acquired pneumonia: results from the German Competence Network, CAPNETZ. Am J Respir Crit Care Med. https://doi.org/10.1164/rccm.201003-0415OC

11. Guertler C, Wirz B, Christ-Crain M et al (2011) Inflammatory responses predict long-term mortality risk in community-acquired pneumonia. Eur Respir J. https://doi.org/10.1183/0903193600 121510

12. Bello S, Lasierra AB, Mincholé E et al (2012) Prognostic power of proadrenomedullin in community-acquired pneumonia is independent of aetiology. Eur Respir J. https://doi.org/10.1183/09031 93600080411

13. Melchio R, Rinaldi G, Testa E et al (2018) Red cell distribution width predicts mid-term prognosis in patients hospitalized with acute heart failure: the RDW in Acute Heart Failure (RE-AHF) study. Intern Emerg Med 14:239-247
14. Salvagno GL, Sanchis-Gomar F, Picanza A, Lippi G (2015) Red blood cell distribution width: a simple parameter with multiple clinical applications. Crit Rev Clin Lab Sci 52:86-105. https:// doi.org/10.3109/10408363.2014.992064

15. Horne BD, Muhlestein JB, Bennett ST et al (2015) Association of the dispersion in red blood cell volume with mortality. Eur J Clin Invest. https://doi.org/10.1111/eci.12432

16. Yčas JW, Horrow JC, Horne BD (2015) Persistent increase in red cell size distribution width after acute diseases: a biomarker of hypoxemia? Clin Chim Acta 448:107-117. https://doi. org/10.1016/j.cca.2015.05.021

17. Tonelli M, Wiebe N, James MT et al (2019) Red cell distribution width associations with clinical outcomes: a populationbased cohort study. PLoS ONE. https://doi.org/10.1371/journ al.pone. 0212374

18. Bello S, Fandos S, Lasierra AB et al (2015) Red blood cell distribution width [RDW] and long-term mortality after community-acquired pneumonia. A comparison with proadrenomedullin. Respir Med 109:1193-1206. https://doi.org/10.1016/j. rmed.2015.07.003

19. Lee S-M, Lee JH, Kim K et al (2016) The clinical significance of changes in red blood cell distribution width in patients with community-acquired pneumonia. Clin Exp Emerg Med 3:139-147. https://doi.org/10.15441/ceem.15.081

20. Moons KGM, Altman DG, Reitsma JB et al (2015) Transparent reporting of a multivariable prediction model for individual prognosis or diagnosis (TRIPOD): Explanation and elaboration. Ann Intern Med. https://doi.org/10.7326/M14-0698

21. Woodhead M, Blasi F, Ewig S et al (2011) Guidelines for the management of adult lower respiratory tract infectionssummary. Clin Microbiol Infect. https://doi.org/10.111 1/j.1469-0691.2011.03602.x

22. Society BT (2009) Guidelines for the Management of Community Acquired Pneumonia in Adults Update 2009 A Quick Reference Guide. Society 64:

23. Levey AS, Stevens LA, Schmid CH et al (2009) A new equation to estimate glomerular filtration rate. Ann Intern Med. https://doi. org/10.7326/0003-4819-150-9-200905050-00006

24. Fine MJ, Auble TE, Yealy DM et al (1997) A prediction rule to identify low-risk patients with community-acquired pneumonia. N Engl J Med. https://doi.org/10.1056/NEJM199701233360402

25. Lim WS, Van Der Eerden MM, Laing R et al (2003) Defining community acquired pneumonia severity on presentation to hospital: an international derivation and validation study. Thorax. https ://doi.org/10.1136/thorax.58.5.377

26. Schuetz P, Christ-Crain M, Thomann R et al (2009) Effect of procalcitonin-based guidelines vs standard guidelines on antibiotic use in lower respiratory tract infections: The ProHOSP randomized controlled trial. JAMA. https://doi.org/10.1001/jama.2009.1297

27. Xue X, Agalliu I, Kim MY et al (2017) New methods for estimating follow-up rates in cohort studies. BMC Med Res Methodol. https://doi.org/10.1186/s12874-017-0436-z

28. Sullivan LM, Massaro JM, D’Agostino RB (2004) Presentation of multivariate data for clinical use: The Framingham Study risk score functions. Stat Med. https://doi.org/10.1002/sim.1742

29. Royston P (2015) Tools for checking calibration of a Cox model in external validation: prediction of population-averaged survival curves based on risk groups. Stata J. https://doi.org/10.1177/15368 $67 \times 1501500116$

30. Royston P, Moons KGM, Altman DG, Vergouwe Y (2009) Prognosis and prognostic research: developing a prognostic model. BMJ. https://doi.org/10.1136/bmj.b604

31. Childs BG, Durik M, Baker DJ, Van Deursen JM (2015) Cellular senescence in aging and age-related disease: from mechanisms to therapy. Nat Med 21:1424-1435 
32. Shivshankar P, Boyd AR, Le Saux CJ et al (2011) Cellular senescence increases expression of bacterial ligands in the lungs and is positively correlated with increased susceptibility to pneumococcal pneumonia. Aging Cell. https://doi.org/10.111 1/j.1474-9726.2011.00720.x

33. Braun E, Domany E, Kenig Y et al (2011) Elevated red cell distribution width predicts poor outcome in young patients with community acquired pneumonia. Crit Care 15:R194. https://doi. org/10.1186/cc10355

34. Braun E, Kheir J, Mashiach T et al (2014) Is elevated red cell distribution width a prognostic predictor in adult patients with community acquired pneumonia? BMC Infect Dis 14:129. https ://doi.org/10.1186/1471-2334-14-129\r1471-2334-14-129[pii]

35. Lee JH, Chung HJ, Kim K et al (2013) Red cell distribution width as a prognostic marker in patients with community-acquired pneumonia. Am J Emerg Med 31:72-79. https://doi.org/10.1016/j. ajem.2012.06.004

36. Hong N, Oh J, Kang SM et al (2012) Red blood cell distribution width predicts early mortality in patients with acute dyspnea. Clin Chim Acta 413:992-997. https://doi.org/10.1016/j. cca.2012.02.024

37. Corrales-Medina VF, Alvarez KN, Weissfeld LA et al (2015) Association between hospitalization for pneumonia and subsequent risk of cardiovascular disease. JAMA 313:264-274. https ://doi.org/10.1001/jama.2014.18229

38. Corrales-Medina VF, Musher DM, Shachkina S, Chirinos JA (2013) Acute pneumonia and the cardiovascular system. Lancet 381:496-505. https://doi.org/10.1016/S0140-6736(12)61266-5

39. Shah N, Pahuja M, Pant S et al (2017) Red cell distribution width and risk of cardiovascular mortality: insights from National
Health and Nutrition Examination Survey (NHANES)-III. Int J Cardiol. https://doi.org/10.1016/j.ijcard.2017.01.045

40. Zalawadiya SK, Veeranna V, Niraj A et al (2010) Red cell distribution width and risk of coronary heart disease events. Am J Cardiol. https://doi.org/10.1016/j.amjcard.2010.06.006

41. Ewig S, Welte T, Chastre J, Torres A (2010) Rethinking the concepts of community-acquired and health-care-associated pneumonia. Lancet Infect Dis 10:279-287

42. Polverino E, Torres A, Menendez R et al (2013) Microbial aetiology of healthcare associated pneumonia in Spain: a prospective, multicentre, case-control study. Thorax. https://doi.org/10.1136/ thoraxjnl-2013-203828

43. Torres A, Niederman MS, Chastre J et al (2017) International ERS/ESICM/ESCMID/ALAT guidelines for the management of hospital-acquired pneumonia and ventilator-associated pneumonia. Eur Respir J 50:1700582

44. Gallo P, De Vincentis A, Pedone C et al (2019) Drug-drug interactions involving CYP3A4 and p-glycoprotein in hospitalized elderly patients. Eur J Intern Med. https://doi.org/10.1016/j. ejim.2019.05.002

45. Mannucci PM, Nobili A, Pasina L et al (2018) Polypharmacy in older people: lessons from 10 years of experience with the REPOSI register. Intern Emerg Med 13:1191-1200

Publisher's Note Springer Nature remains neutral with regard to jurisdictional claims in published maps and institutional affiliations. 